\title{
Evolving coronary bifurcation intervention techniques. Can „double kiss" change our routine?
}

\author{
Viktor Sasi, Gyula Szántó, Attila Thury, András Katona, Róbert Sepp, \\ Attila Nemes, Imre Ungi
}

Division of Invasive Cardiology, Department of Medicine, Medical Faculty, Albert Szent-Györgyi Clinical Center, University of Szeged, Szeged, Hungary

Address for correspondence: Viktor Sasi, MD, PhD, Division of Invasive Cardiology,

Albert Szent-Györgyi Clinical Center, University of Szeged, Hungary, H-6725 Szeged, Semmelweis str. 8.

E-mail: sasiviktor@gmail.com, sasi.viktor@med.u-szeged.hu

In the coronary tree, there are several major coronary side branch ostia. If the daughter vessels are estimated to supply $10 \%$ of the myocardium then they could be considered as not negligible vessels. In the past 25 years, there have been several efforts to describe and classify the "coronary bifurcation". In the past decade, a consensus has been reached by the European Bifurcation Club to use the "Medina Classification" for description of true bifurcation lesions. To describe the treatment options for a true bifurcation the MADS classification has been proposed. The European Bifurcation Club insists on supporting the so called "provisional stenting" technique, but lately with the development of new generation devices the "2-stent strategy" has evolved and shows convincing long term results, thus for complex anatomy it could be the first line planned strategy for treatment.

Keywords: coronary atherosclerosis, coronary bifurcation, percutaneous coronay intervention

\section{Introduction}

A simple description of a bifurcating vessel is not easy. There have been several publications on different descriptions of bifurcations (1). In the last decade the consensus has been reached that the Medina classification would suit most of the bifurcating vessels and it is fairly simple to describe bifurcations $(2,3)$ (Figure 1). The functional importance of the side branch and supplied myocardium could be debated.

\section{Proper angiographic assessment}

Due to the three dimensional structure of a bifurcation it is not possible to avoid foreshortening of originating vessel, thus it is necessary to have multiplane recordings of the three parts of the bifurcation to have comprehensive images to best describe lesion characteristics. Most of the time the origin of the side branch (SB) could only be described from at least two orthogonal projections. Optimal image of the SB ostium must include perpendicular view to the ostium of the SB. Increasing number of elective patients have undergone coronary CT acquisition before the intervention (PCI). If CT scan is at hand, then three dimensional CT images could help in identifying the extent of plaque in the three parts of the bifurcation and finding the best optimal "working view". Three-dimensional (3D) quantitative coronary analysis (QCA) images could be generated to also help procedural planning (4). 


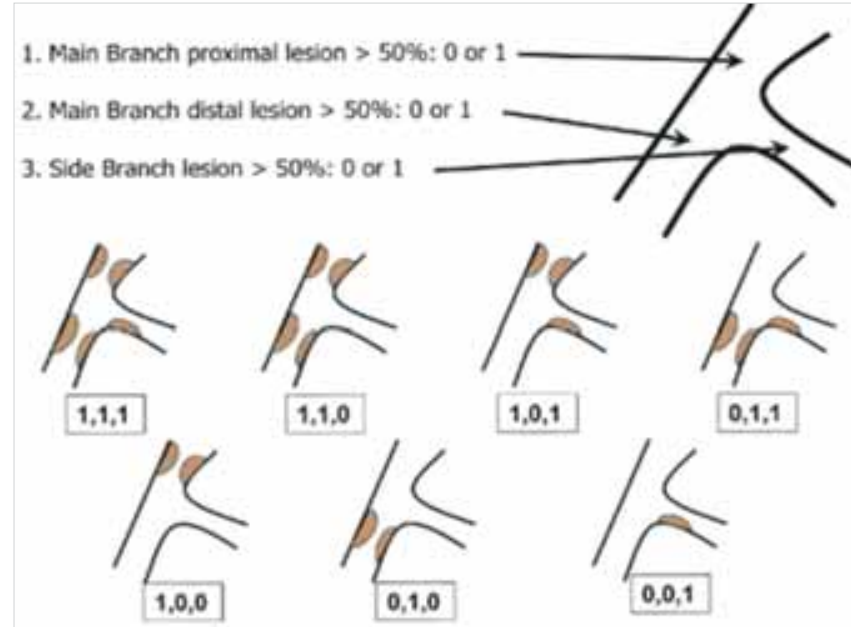

FIG. 1. Medina Classification of coronary bifurcation lesions (Medina A, Suarez de Lezo J, Pan M. [A new classification of coronary bifurcation lesions]. Rev Esp Cardiol. 2006; 59: 183.)

\section{The importance of the side branch}

The importance of the supplied territory of the side branch remains a question of debate. In one publication coronary CT and fractional flow reserve (FFR) was used to determine the SB vessel importance and it was found that 73 millimeter length was supplying at least

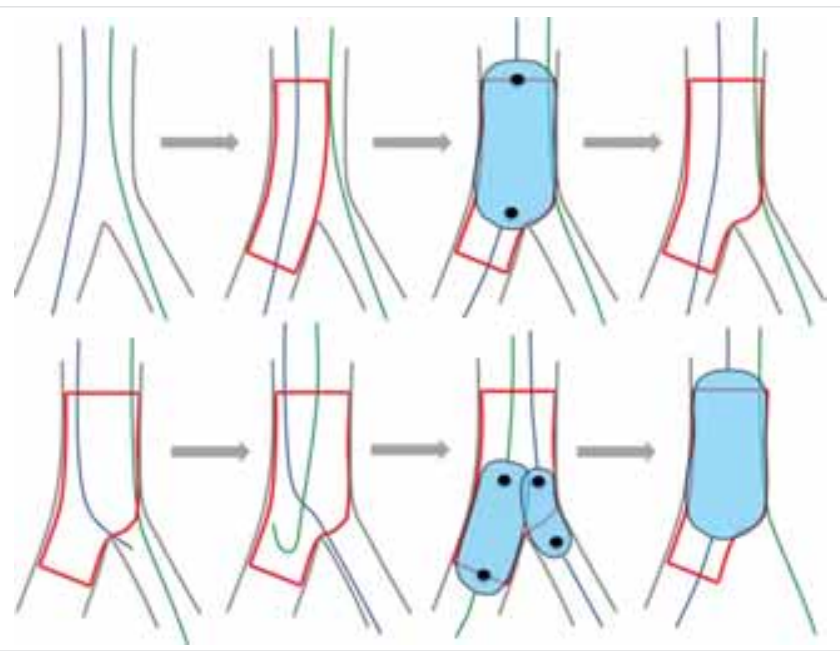

FIG. 2. Summary of the provisional approach. Upper panel from left to right: two wires in place, main branch stent sized according to the distal reference, POT, keep it open. Lower panel: access towards the distal strut, guidewire exchange, kissing balloon inflation with short non-compliant balloons (not proximal to the polygon of confluence to avoid dilating the distal part of the stent too much towards the side branch). A final POT should be carried out if the two balloons are proximal to the polygon of confluence.

Jens Flensted Lassen et al. Percutaneous coronary intervention for the left main stem and other bifurcation lesions: $12^{\text {th }}$ consensus document from the European Bifurcation Club, Eurolntervention 2018; 13: 1540-1553.
$10 \%$ of myocardium territory (5). It is expected that the first diagonal and circumflex or obtus marginal would have the biggest territory to supply when treated as a side branch. The length of the SB and the degree of plaque causing significant stenosis in the side branch ( $\leq 5 \mathrm{~mm}$ from the ostium) would highly constitute to determining the functional significance of the SB supplied territory. In these cases or if anatomical obstacles or long protruding plaque to the SB is present it would decrease the potential of rewiring after main branch (MB) stenting, so it is worth to upfront think about 2-stent strategy or at least to place a safety wire into the side branch (6).

\section{Provisional bifurcation stenting strategy}

The provisional SB treating strategy is currently considered as the standard of care in $\mathrm{PCl}$ involving significant bifurcation lesions. This strategy could be considered as the "keep-it-open" strategy $(7,8)$ (Figure 2). First the MB should be identified, after that both branches should be wired. The MB then should be stented sized according to the MB distal vessel size. This should be followed by proximal optimization (POT) by a balloon sized to the proximal stented segment. Noncompliant balloon (NC balloon) use is not mandatory, but recommended. After POT the SB ostium should be evaluated. If the SB requires further treatment, guidewires could be exchanged and kissing balloon inflation (KBI) or POT/side/POT (re-POT) could be carried out $(9,10)$. In the presence of SB flow major disturbance or poor angiographic result, SB stenting could be performed. T stenting, $T$ and protrusion (TAP) or Culotte with systematic final KBI and POT should be taken into consideration (10) (Figure $3 A, B)$.

\section{Proximal optimization technique}

New generation drug eluting stents (DES) can be adapted to the fractal anatomy of bifurcation lesions by POT, especially in bifurcation lesions where a large SB is taking off and the angle of take-off is not too steep (10). For the most ideal POT the MB stent proximal to the carina should have at least $6-10 \mathrm{~mm}$ of stent length to facilitate a short balloon sized to the proximal MB reference diameter $(7,10)$. Careful positioning of the balloon for proximal optimization is critical. For proper proximal placement of the Compliant/NC balloon for POT there are stent enhancement techniques available. There is data suggesting that SB ostium closure after POT is a real issue, thus to avoid carina shift towards the SB it is critical to place the distal edge of the balloon to the polygon of confluence, but not further. The balloon to proximal MB vessel ratio for 
A

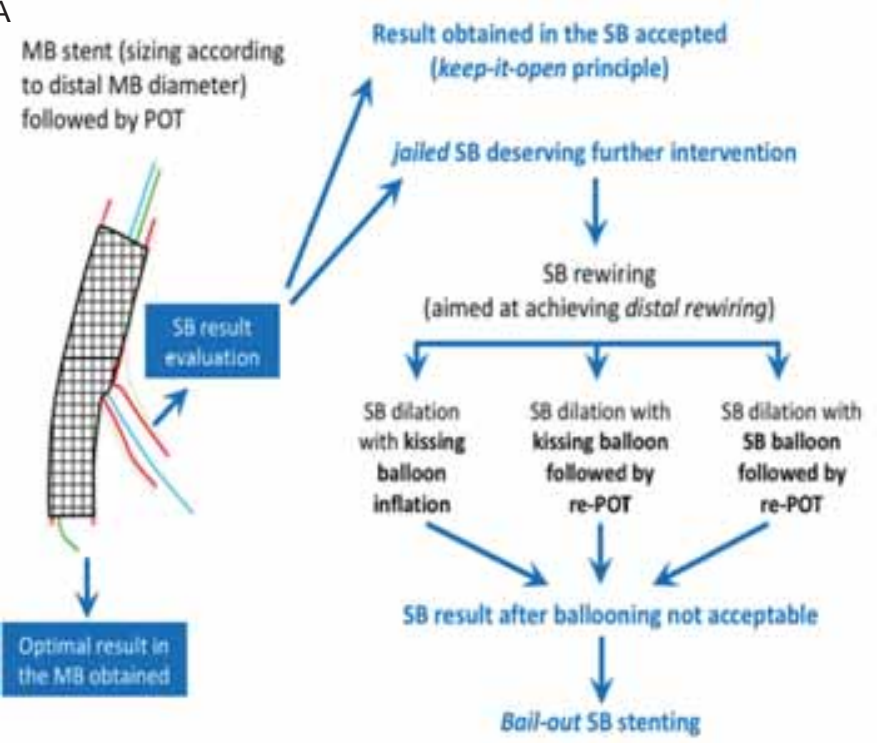

B

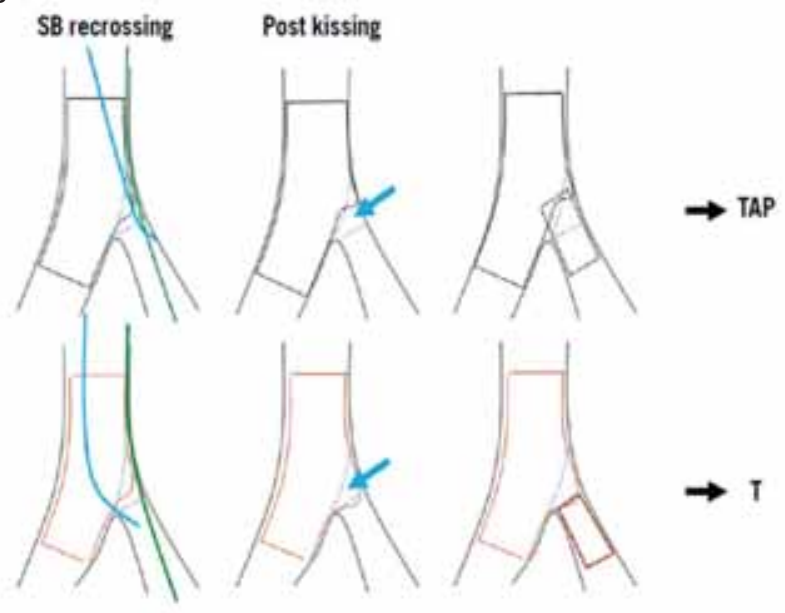

FIG. 3. The philosophy of the provisional bifurcation stenting approach. From left to right: main branch stenting with stent implantation that respects the distal main branch diameter, finalised with POT to respect the original tapering anatomy and to open the struts towards the side branch. If the side branch is compromised, continue to dilatation of the side branch. If still compromised, an escalation to side branch stenting is facilitated. Always finalise a procedure with POT.

Banning AP, Lassen JF, Burzotta F et al. Percutaneous coronary intervention for obstructive bifurcation lesions: the $14^{\text {th }}$ consensus document from the European Bifurcation Club. Eurolntervention. 2019 May 20; 15 (1): 90-98

POT should not exceed $1 / 1$. Now it is recommended to use NC balloons for POT. NC balloons hold shape better, thus overhanging balloon and dissection of the proximal MB non-covered segment is less frequent, furthermore the SB ostium dissection is less frequent (11).

\section{Side branch opening}

In the majority of cases provisional stenting is the most suitable, less time and equipment consuming option (7). If the SB originating from the MB is considered to be important a wire should always be placed in the vessel. If after MB stenting and POT the ostium of the SB is compromised either the MB wire should be placed in the side branch, but if the MB stenting result is not satisfactory or more lesions should be treated, then a third, new wire should be placed in the SB. First the side branch ostium stent covered struts should be opened with a balloon. The balloon to vessel ratio of maximum 0.8 should be reached. After this a final repeated POT should be carried out. If the result is not satisfactory $\mathrm{KBI}$ and POT should be performed to minimize eccentricity index $(12,7)$. At this point a good quality image from multiple projections is necessary to evaluate the achieved result and potentially finish the procedure with one stent. As for now the EBC recommends POT/KBI/ POT for less stent deformity in case of one stent technique (7) (Figure 3A).

\section{In case of unsatisfactory result after KBI and re-POT}

The use of second stent could be needed in $10-25 \%$ of cases started as the provisional approach. If after POT/ $\mathrm{KBI} / \mathrm{POT}$ the result is unsatisfactory or flow is decreased a second stent should be placed in the side branch. T or $\mathrm{T}$ and Protrusion (TAP) or Culotte stenting could be performed in this case. Generally, T stenting could only be an option after stent enhancement if the SB wire crossed in a distal position and POT was performed ending at the polygon of confluence. In this case the stent has been opened up and there is no need to create a neocarina. Even in this case careful positioning of the second stent is advised not to leave a gap (Figure 3B). In most cases TAP technique is advised, which creates a small neocarina in the ostium of the $\mathrm{SB}$, thus ensures perfect coverage of the $3 D$ structure of the bifurcation (Figure $3 B$ ). In both cases the procedure should be finished with $\mathrm{KBI}$ and POT (7). If there is a fear of not covering the ostium of the SB perfectly with TAP because of the motion of the vessel or complex anatomy and calcium presence Culotte should be chosen $(7,19)$.

\section{Planned Two-stent strategy}

If a high complexity bifurcation is observed an upfront 2-stent strategy should be considered. Other indications for planned 2-stent strategy is the significant calci- 

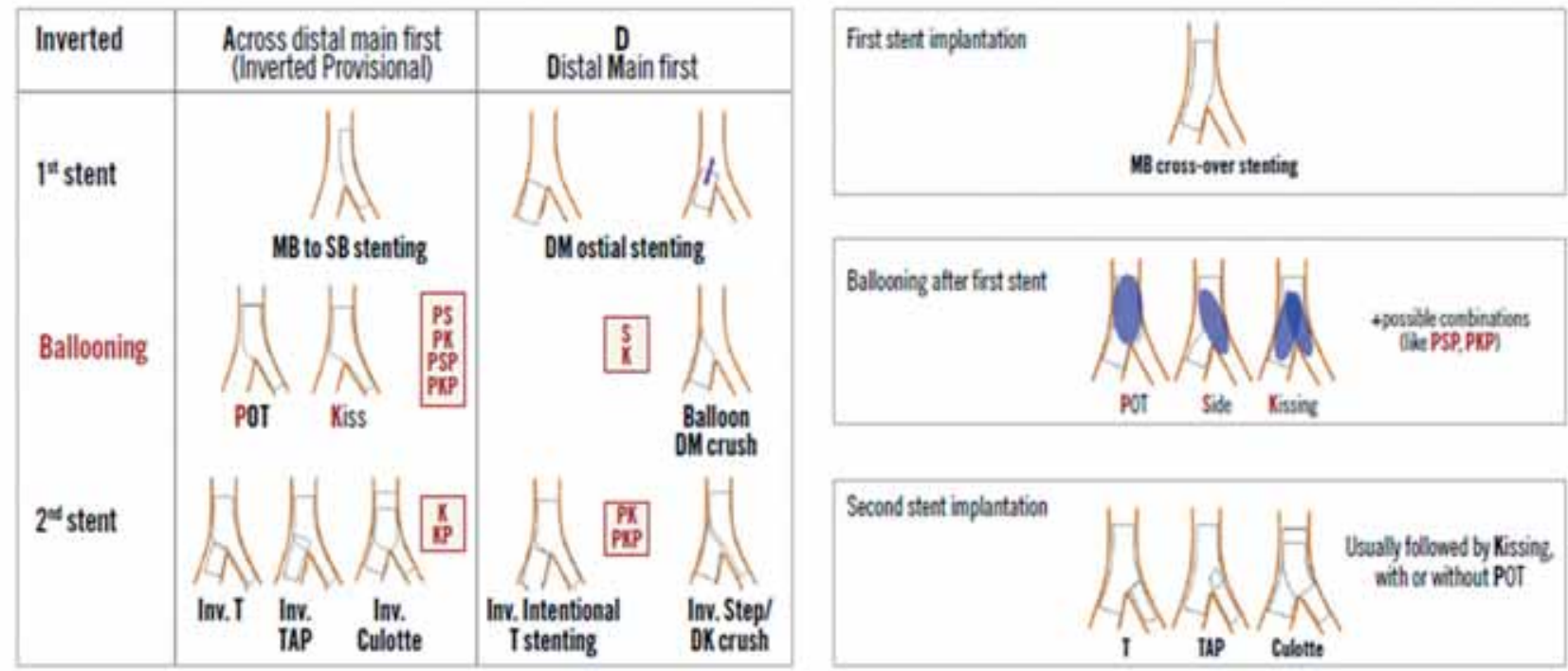

FIG. 4. The new amended MADS (2019) classification. Three types of balloon dilation techniques are considered pivotal during bifurcation treatment. These have been inserted in MADS-2 using a single letter ballooning technique code: $\mathrm{P}=$ post-dilation of the proximal main vessel (usually reported as proximal optimisation technique [POT]); $\mathrm{S}=$ balloon dilation of the SB ostium; $\mathrm{K}=$ balloon inflation in the MV and SB (usually reported as kissing balloon inflation technique). Combinations of the ballooning techniques can be highlighted by appropriately ordered letter sequences, e.g. PKP stands for POT, followed by kissing, followed by further POT. PSP represents POT, followed by side branch inflation, followed by final POT.

Banning AP, Lassen JF, Burzotta F et al. Percutaneous coronary intervention for obstructive bifurcation lesions: the $14^{\text {th }}$ consensus document from the European Bifurcation Club. Eurolntervention. 2019 May 20; 15 (1): 90-98.

um in the SB ostium and the significant atherosclerotic disease protruding to the SB more than $5 \mathrm{~mm}$. In all of these cases the proper lesion preparation is crucial for good stent apposition and scaffolding and most achievable lumen gain (7). Whatever 2-stent strategy is opted the $\mathrm{KBI}$ and POT must follow.

\section{MADS classification}

MADS classification (main/across/distal/side) is based on the final position of the stents in the bifurcation and the sequence in which stents are implanted according to the strategy planned (Figure 4). The position of the first stent in a bifurcation corresponds to a given strategy (13) 7). M begins by a stent in the proximal MB segment. A starts with a stent in the MB across the SB. D is for Double stent implantation whether simultaneous or not. S strategy consists of a stent implantation in the SB first with protrusion (short or long) or not $(13,7)$. The application of the MADS classification in a large stent trial showed, that $80.3 \%$ of bifurcations were treated using a single stent, $18.9 \%$ using 2 stents and $0.7 \%$ using 3 stents. Overall, the "main across side first" strategy (A) was used in $77.4 \%$ with the "side branch first" approach (S) being the second most frequently used technique $(10.2 \%)(14)$.

\section{Crush}

If planned 2-stent strategy is an option, then a modification of the "Crush" technique always must be considered. The previously mentioned "original Crush" technique by Colombo was published in 2003 (15). The low percentage of rewiring rate of the SB, thus lack of final $\mathrm{KBI}$ and POT resulted in unfavorable long-term outcome and MACE rate, thus it is not recommended anymore. From then it has come a long way to become the number one strategy for 2-stent technique even in unprotected left main lesions (7). The steps of the original Crush technique have been improved by Chen and colleagues. It has become a more complex procedure, since after crushing the first stent towards the SB it requires POT in the MB with careful positioning of the POT balloon and KBI. This change to the original procedure brought dramatic rise in the $S B$ rewiring success after the second stent implantation in the MB three involved parts, thus the success of carrying out of the $2^{\text {nd }} \mathrm{KBI}$ and re-POT jumped to above $90-100 \%$ in experienced hands (16). In the DKCRUSH-II study this strategy was directly compared to the provisional stenting strategy and target lesion revascularization (TLR) and target vessel revascularization (TVR) showed better results, but MACE rate showed no difference (17). There was also a lot of criticism expressed towards this study for various reasons. 


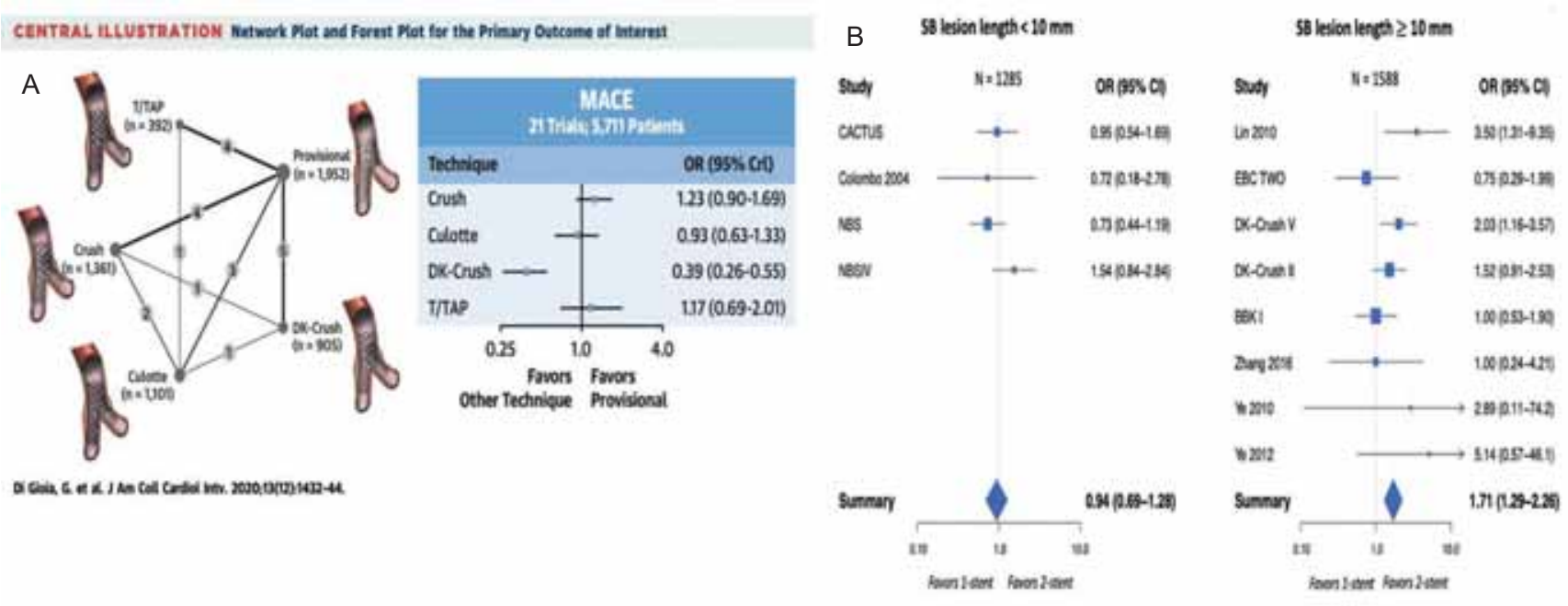

FIG. 5. A: Left: The figure is showing the number of patients included in the studies with different techniques. Right: If only MACE is taken into consideration it would favor the 2-stent techniques, amongst them DK-CRUSH shows the best results.

Giuseppe Di Gioia, Jeroen Sonck, Miroslaw Ferenc et al. Clinical Outcomes Following Coronary Bifurcation PCI Techniques A Systematic Review and Network Meta-Analysis Comprising 5,711 Patients JACC: Cardiovascular Interventions Volume 13, Issue 12, June 2020.

B: Pairwise Meta-Analysis of the Outcome of MACE Between 1- and 2-Stent Bifurcation PCI Strategies Stratified According to SB Lesion Length (Left) Forest plot with studies reporting side branch (SB) lesion length $<10 \mathrm{~mm}$. The summary estimate shows no difference between 1- and 2-stent bifurcation percutaneous coronary intervention (PCl) strategies. (Right) Forest plot with studies reporting SB lesion length $\geq 10 \mathrm{~mm}$. The summary estimate favors 2-stent bifurcation $\mathrm{PCl}$ techniques.

Giuseppe Di Gioia, Jeroen Sonck, Miroslaw Ferenc et al. Clinical Outcomes Following Coronary Bifurcation PCI Techniques A Systematic Review and Network Meta-Analysis Comprising 5,711 Patients JACC: Cardiovascular Interventions Volume 13, Issue 12, June 2020

\section{Culotte}

The original Culotte stenting technique was described by Chevalier in 1998 (18). At that time a new strategy was described to protect the SB of a true bifurcation from short or long term closure. The implantation of 2 fairly similar stents in the MB and in the SB with overlapping of the 2 stents in the MB proximal to the bifurcation part was proposed. This strategy has several potential advantages over the other techniques. The intervention can be carried out as a provisional approach if only one stent is implanted but there is the option to proceed and switch to 2-stent approach. One potential advantage over the Crush technique of having only two and not three stent layers in the proximal part of the bifurcation lesion, potentially leading to a lower risk of stent malapposition. Rewiring of the SB and KBI could be easier than with the Crush technique. The potential of better bifurcation coverage and thus having lower rate of recoil rate and residual restenosis has to be measured. In an angiographic follow up large scale analysis with the use of $\mathrm{KBI}$ restenosis occurred in $15.5 \%$ of patients with vs. $33.3 \%$ of patients without KBI and POT (19). Also in this analysis the predictors for angiographic restenosis were older age, increasing bifurcation angle (BA), more severe stenosis in the distal MB and a smaller reference diameter of the SB (19).

\section{Double Kiss Crush versus Culotte}

Since Culotte and DK-Crush showed satisfying data on long-term outcome for true bifurcation treatment in the DKCRUSH-III trial their performance was compared in the unprotected left main group. In this analysis the Culotte group showed significantly higher 1-year MACE rate $(16.3 \%)$, mainly driven by increased TVR $(11.0 \%)$, compared with the DK-Crush group $(6.2 \%$ and $4.3 \%$, respectively; all $\mathrm{P}<0.05)$. Definite stent thrombosis rate was not significant (20).

\section{Comparison of randomized controlled trials of different bifurcation techniques}

In a recent meta-analysis of a large scale of patients provisional stenting, T stenting/T and protrusion, Crush, Culotte, and DK-Crush was investigated. Median follow-up duration was 12 months. MACE rate was the least in DK-Crush, it was mainly driven by TLR (OR: 0.39 ; $95 \%$ credible interval: 0.26 to 0.55 vs. OR: 0.36 ; 95\% credible interval: 0.22 to 0.57 ) (Figure 5A). Cardiac death, myocardial infarction and stent thrombosis showed no significant difference (21). Clinical benefit was observed in the 2-stent techniques where the SB lesion showed $10 \mathrm{~mm}$ of length from the ostium (Figure 
A

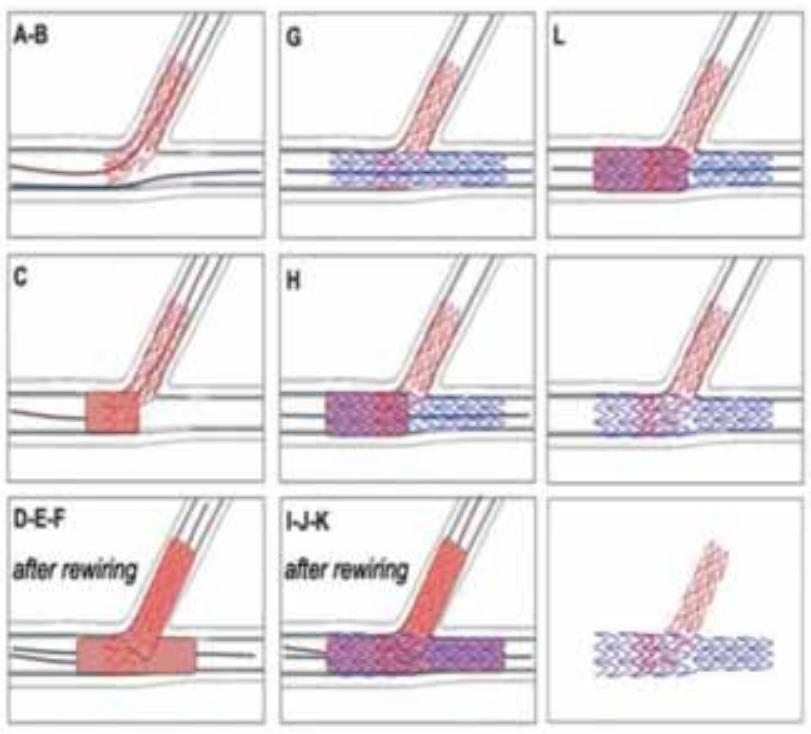

$\mathrm{B}$
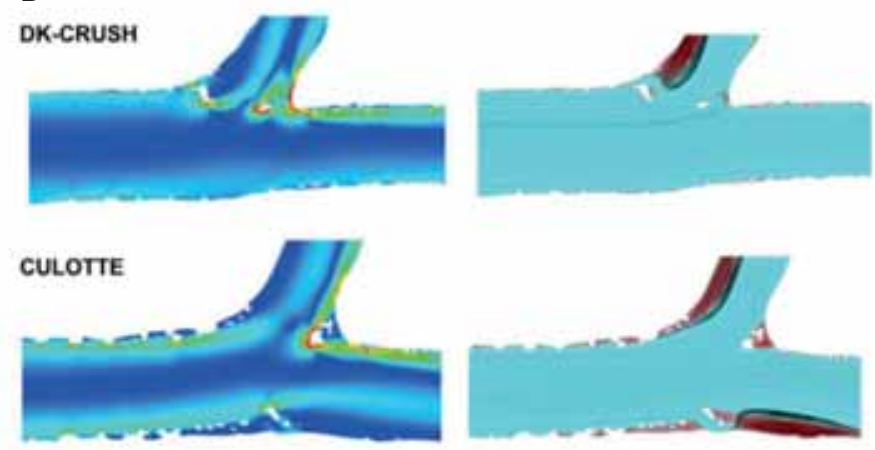

DK-CULOTTE
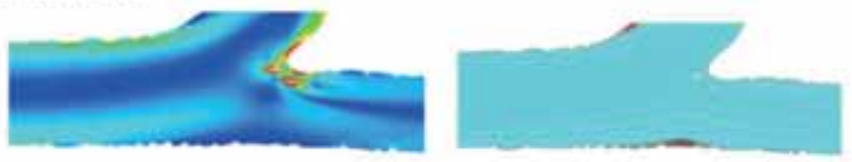

FIG. 6. A:The major steps of the DK-Culotte technique from A-L and outcome Toth GG, Sasi V, Franco D et al. Double-Kissing Culotte Technique for Coronary Bifurcation Stenting - Technical evaluation and comparison with conventional double stenting techniques. Eurolntervention. 2020 Apr 28

B: Representative cases for computational fluid dynamic analysis of DK-Crush, Culotte and DK-Culotte procedures. Toth GG, Sasi V, Franco D et al. Double-Kissing Culotte Technique for Coronary Bifurcation Stenting - Technical evaluation and comparison with conventional double stenting techniques. Eurolntervention. 2020 Apr 28

$5 B)$. What can be clearly seen, that the original crush was greatly improved by the introduction of the double kiss technique (21). Conventional Culotte shows better results than provisional stenting if MACE is taken into consideration (Figure 5A).

\section{From Culotte to Double Kiss Culotte}

The hypothetical advantages of the Culotte technique are several. First, clinically most important, that it allows to start the bifurcation $\mathrm{PCl}$ using the provisional approach. Second, no stent is designed to be crushed, thus in the proximal MB there is only two and not three stent layers, which does have a hemodynamic effect, thus the chance of proper stent apposition is better (19). In the first major publication of the Culotte long term follow up showed some interesting data (19). Adriaenssens et al. in 2008 only used "first generation DES" for their analysis. Only in $62 \%$ of the cases KBI was performed. In this analysis considering the above mentioned details only $16 \%$ of the cases binary restenosis was observed at the SB ostium. MB proximal part showed $0 \%$ of ISR, distal MB showed $9 \%$ of ISR at 12-month (19). 52 o bifurcation angle was the cut off value for higher rate of restenosis in the SB (19). With respect to this publication's data and other meta-analysis (21) it is obvious that the conventional Culotte can only be improved by the introduction of the double kiss technique (Figure 6A) (22). Nowadays with new generation DES the only potential problem to take into consideration is the major caliber mismatch between the $\mathrm{SB}, \mathrm{MB}$. This is where Culotte/DK-Culotte could lose some ground to favor the DK-Crush technique.

In the evolution of the different bifurcation techniques there is a new publication of Tóth, Sasi et al. performing a bench test analysis directly comparing Culotte, DK-Culotte and DK-Crush for stent apposition, for luminal opening and for flow dynamics. In this analysis total procedure duration of DK-Culotte was significantly lower than for DK-Crush, however similar to Culotte (22). In DK-Culotte overall rate of moderate $(200-500 \mu \mathrm{m})$ and significant $(>500 \mu \mathrm{m})$ malapposition was similar as compared to Culotte and lower as compared to DK-Crush. Lower malapposition rate of DK-Culotte as compared to DK-Crush was due to less moderate and significant malapposition in proximal MB (22). Micro computed tomography did not show difference in luminal opening at the site of the proximal MB, distal MB or SB. There was no difference either in maximum shear rate or in areas of high shear or recirculation (22) (Figure 6 B). This new data would suggest that the above mentioned potential advantages could have potential clinical advantages and DK-Culotte could have even better long term results than DK-Crush, although this hypothesis has to be confirmed with large scale patient data from randomized controlled trails. 


\section{Conclusion}

The vast majority of the non-complex coronary bifurcation cases can and should be solved with the provisional technique since it is easier and faster to carry out. If something unsatisfactory happens to the SB then the procedure could be transformed to a 2-stent procedure namely TAP or Culotte. If a complex anatomy is encountered or major calcification is present or long lesion is present at the SB ostium or more distally, then an upfront 2-stent strategy should be opted. In the evolution process of the planned 2-stent techniques the double-kiss approach facilitates long term good outcome. DK-Crush and DK-Culotte should be opted if planned 2-stent strategy is chosen. DK-Culotte has some theoretical and proven bench test advantages over DK-Crush, but further real world data is needed to support bench test data.

\section{Declaration of interest}

The author has reported that he has no relationships relevant to the contents of this paper to disclose.

\section{References}

1. Lefèvre $T$, Louvard $Y$, Morice $M C$, et al. Stenting of bifurcation lesions: Classification, treatments, and results. Catheter Cardiovasc Interv 2000; 49: 274-283. DOI: 10.1002/(sici)1522726x(200003)49:3<274::aid-ccd11>3.0.co;2-n

2. Medina A, Suarez de Lezo J, Pan M. A new classification of coronary bifurcation lesions. Rev Esp Cardiol 2006; 59: 183. DOI: 10.1016/S1885-5857(06)60130-8

3. Louvard Y, Thomas M, Dzavik V, Hildick-Smith D, et al. Classification of coronary artery bifurcation lesions and treatments: time for a consensus! Catheter Cardiovasc Interv 2008; 71: 175-83. DOI: $10.1002 / \mathrm{ccd} .21314$

4. Tu S, Hao P, Koning G, et al. In vivo assessment of optimal viewing angles from X-ray coronarography. Eurolntervention 2011; 7: 112-20. DOI: 10.4244/EIJV7I1A19

5. Uzo K, Otake H, Choi G, et al. Lumen boundaries extracted from coronary computed angiography on computed fractional flow reserve (FFRCT): validation with optical coherence tomography. Eurolntervention 2019; 14e: 1609-18. DOI: 10.4244/EIJ-D-17-01132

6. Kim HY, Doh JH, Lim HS, et al. Identification of coronary side branch supplying myocardial mass that may benefit from revascularization. JACC Cardiovascular interventions 2017; 10 : 571-81. DOI: 10.1016/j.jcin.2016.11.033

7. Banning AP, Lassen JF, Burzotta F et al. Percutaneous coronary intervention for obstructive bifurcation lesions: the $14^{\text {th }}$ consensus document from the European Bifurcation Club. Eurolntervention 2019 May 20; 15(1): 90-98. DOI: 10.4244/EIJ-D-19-00144

8. Hildick-Smith D, Behan MW, Lassen JF, et al. The EBC TWO Study (European Bifurcation Coronary TWO): A Randomized Comparison of Provisional T-Stenting Versus a Systematic 2 Stent Culotte Strategy in Large Caliber True Bifurcations. Circ Cardiovasc Interv 2016 Sep; 9(9). DOI: 10.1161/CIRCINTERVENTIONS.115.003643
9. Mortier P, Hikichi Y, Foin N, et al. Provisional stenting of coronary bifurcations: insights into final kissing balloon post-dilation and stent design by computational modeling. JACC Cardiovasc Interv 2014; 7: 325-33. DOI: 10.1016/j.jcin.2013.09.012

10. Finet G, Derimay F, Motreff $P$, et al. Comparative Analysis of Sequential Proximal Optimizing Technique Versus Kissing Balloon Inflation Technique in Provisional Bifurcation Stenting: Fractal Coronary Bifurcation Bench Test. JACC Cardiovasc Interv 2015; 8: 1308 17. DOI: 10.1016/j.jcin.2015.05.016

11. Hoye A. The Proximal Optimisation Technique for Intervention of Coronary Bifurcations. Interv Cardiol 2017; 12: 110-5. DOI: 10.15420/icr.2017:11:2

12. Derimay F, Souteyrand G, Motreff, et al. Influence of platform design of six different drug-eluting stents in provisional coronary bifurcation stenting by rePOT sequence: a comparative bench analysis. EuroIntervention 2017; 13: e1092-5. DOI: 10.4244/EIJ-D-16-00863 13. Yves Louvard, Alfonso Medina. Definitions and classifications of bifurcation lesions and treatment. Eurolntervention 2015; 11: V23V26. DOI: 10.4244/EIJV11SVA5

14. Athanasios Katsikis, Ply Chichareon, Rafael Cavalcante, et al Application of the MADS Classification System in a "Mega Mammoth" Stent Trial: Feasibility and Preliminary Clinical Implications. Catheter Cardiovasc Interventions 2019 Jan 1; 93(1): 57-63.

15. Colombo A, Stankovic G, Orlic D, et al. Modified T-stenting Technique With Crushing for Bifurcation Lesions: Immediate Results and 30-day Outcome. Catheter Cardiovasc Interventions 2003 Oct; 60(2): 145-51. DOI: 10.1002/ccd.10622

16. Chen SL, Zhang JJ, Ye F, et al. Study comparing the double kissing (DK) crush with classical crush for the treatment of coronary bifurcation lesions: the DKCRUSH-1 Bifurcation Study with drug-eluting stents. Eur J Clin Invest 2008; 38: 361-71. DOI: 10.1111/j.1365-2362.2008.01949.x

17. Chen SL, Santoso T, Zhang JJ, et al. A Randomized Clinical Study Comparing Double Kissing Crush With Provisional Stenting for Treatment of Coronary Bifurcation Lesions. Journal of the American College of Cardiology 2011; 5(8). DOI: 10.1016/j.jacc.2010.10.023

18. Chevalier B, Glatt B, Royer T, Guyon P. Placement of coronary stents in bifurcation lesions by the "culotte" technique. Am J Cardiol 1998; 82: 943-949. DOI: 10.1016/s0002-9149(98)00510-4

19. Tom Adriaenssens, Robert A. Byrne, Alban Dibra, et al. Culotte stenting technique in coronary bifurcation disease: angiographic follow-up using dedicated quantitative coronary angiographic analysis and 12-month clinical outcomes European Heart Journal 2008 December; 29(23): 2868-2876. DOI: 10.1093/eurheartj/ehn512

20. Chen SL, Xu B, Han YL, et al. Comparison of Double Kissing Crush Versus Culotte Stenting for Unprotected Distal Left Main Bifurcation Lesions: Results From a Multicenter, Randomized, Prospective DKCRUSH-III Study. Journal of the American College of Cardiology 2013 April 9; 61(14): 1482-1488. DOI: 10.1016/j. jacc.2013.01.023

21. Giuseppe Di Gioia, Jeroen Sonck, Miroslaw Ferenc, et al. Clinical Outcomes Following Coronary Bifurcation PCl Techniques A Systematic Review and Network Meta-Analysis Comprising 5,711 Patients. JACC Cardiovascular Interventions 2020 June; 13(12): DOI: 10.1016/j.jcin.2020.03.054

22. Toth GG, Sasi V, Franco D, et al. Double-Kissing Culotte Technique for Coronary Bifurcation Stenting - Technical evaluation and comparison with conventional double stenting techniques. Eurolntervention 2020 Apr 28: EIJ-D-20-00130 DOI: 10.4244/EIJD-20-00130 